กORTH CLIn ISTARBUL 2015;2(1):81-86

doi: $10.14744 /$ nci.2015.97269

\title{
An overview of Ebola virus disease
}

\author{
Ayten Kadanali, Gul Karagoz \\ Department of Infectious Diseases and Clinical Microbiology, Umraniye Training and Research Hospital, Istanbul, Turkey
}

\begin{abstract}
Ebola virus disease (EVD), formerly known as Ebola hemorrhagic fever, is a severe, often fatal illness in humans. Ebola virus (EBOV) is transmitted through contact with blood or body fluids of a person who contracted or died from EVD, contaminated objects like needles and infected animals or bush meat. EVD has an incubation period of 2 to 21 days, and the infection has an acute onset without any carrier status. Currently, there is no standard treatment for EVD, so it is important to avoid infection or further spreading of the virus. Although historically the mortality of this infection exceeded $80 \%$, modern medicine and public health measures have been able to lower this figure and reduce the impact of EBOV on individuals and communities. Its treatment involves early, aggressive supportive care with rehydration. Clinicians should consider the possibility of EVD in persons with travel or exposure history with the incubation period presenting constitutional symptoms in order to promptly identify diseased patients, and prevent further spreading of the disease.
\end{abstract}

Key words: Ebola virus disease; epidemiology; diagnosis; treatment.

E bola virus disease (EVD) first appeared in 1976 in 2 simultaneous outbreaks in Nzara, Sudan, and in Yambuku, Democratic Republic of Congo. The latter occurred in a village near the Ebola River, from which the disease takes its name. The mortality rate was around 90 percent. Although medical facilities have improved over the years, case fatality rates have varied from $25 \%$ to $90 \%$ in past outbreaks. EVD outbreak that began in February 2014 in Guinea, is the most prevalent EVD outbreak recorded in the history which spred to Liberia, Sierra Leone, Nigeria, Senegal, Spain, and the United States. It had common features with the outbreak in 1976. Zaire Ebola virus was isolat- ed in both outbreaks with the disease onset in rural forest communities. The outbreaks were worsened because critically ill patients with severe systemic symptoms were brought to hospitals which infected hospital staff was unaware of the risks of being exposed to patient blood and body fluids without proper protection. Due to the common travel and general international contacts implicit in globalization, as well as the popularity of tourism, EVD is most certainly a threat to the people all over the world $[1,2,3,4]$. In this review article, we planned to discuss the epidemiological, clinical and laboratory features and patient management of EVD based on literature.

Revised: February 06, 2015 Accepted: April 09, 2015 Online: April 24, 2015

Correspondence: Dr. Ayten KADANALI. Umraniye Egitim ve Arastirma Hastanesi, Enfeksiyon Hastaliklari Bolumu, Istanbul, Turkey.

Tel: +90 216 - 6321818 / 1675 e-mail: ayten.kadanali@gmail.com

(c) Copyright 2015 by Istanbul Northern Anatolian Association of Public Hospitals - Available online at www.kuzeyklinikleri.com 


\section{Virology}

The virus family Filoviridae includes 3 genera: Cuevavirus, Marburgvirus, and Ebolavirus (EBOV). There are 5 species that have been identified: Zaire, Bundibugyo, Sudan, Reston and Tai Forest in Ebolavirus. Zaire, Bundibugyo, and Sudan ebolaviruses have been associated with large outbreaks in Africa. The virus causing the 2014 West African outbreak belongs to the Zaire species. Fruit bats of the family Pteropodidae are the recognized reservoir of the virus. The EBOV genome is a negative-sense single-stranded RNA and contain viral envelope, matrix, and nucleocapsid components. It encodes seven structural proteins: nucleoprotein (NP), polymerase cofactor (VP35, VP40, GP), transcription activator (VP30, VP24), and RNA-dependent RNA polymerase (L) [1]. EBOV is listed as an agent included in WHO Risk Group 4 Pathogen requiring biosafety level 4-equivalent containment requiring Biosafety Level 4-equivalent containment, because of its high mortality rate.

\section{Epidemiology}

The initial outbreak of EVD occurred in Yambuku in the Northern Zaire. Disease had spred by close personal contact. This was the first recognition of the disease and the subtype was named Zaire Ebola Virus [2]. In the same year, another unrelated virus, which resulted in epidemics in South Sudan, was identified as Sudan Ebola Virus [3]. Of interest, some researchers suggested that EBOV outbreaks might be related to certain confluences of environmental and climatic conditions [4].

Up to date, 25 outbreaks have been reported including the current outbreak $(n=4,1976-1979$; $\mathrm{n}=6,1994-1996 ; \mathrm{n}=9,2000-2008$, and $\mathrm{n}=6$ after $2011[5,6]$. Current outbreak has been the largest documented outbreak of EVD and involved the Zaire species of the virus. The outbreak has been mostly seen in Guinea, Liberia and Sierra Leone, which together account for over $99 \%$ of all cases [7]. It is indicated that the exponential growth phase is over in Liberia, with an expected final attack rate of $\sim 0.1-0.12$ percent [8]. The natural reservoir of Ebola virus has not been identified yet. However, it is believed that the first patient became infected through contact with an infected animal like a fruit bat or nonhuman primate. When an infec- tion occurs in humans, the virus can be transmitted through direct contact with blood, body fuids of a EVD patient, needles or syringes that have been contaminated with the virus or infected fruit bats or primates. Ebola virus infection does not spread through contaminated air water, food, or by mosquito and other insects bites.

Once people recover from Ebola, they can no longer spread the virus to people through conventional routes. For instance, men who have recovered from the disease can still transmit the virus through their semen for up to 7 weeks after recovery from illness.

One EVD outbreak started in Guinea in late 2013 which was confirmed by the World Health Organization (WHO) in March 2014. As of March, 18 2015, 24.754 suspect cases and 10.236 deaths had been reported [9]. The case-fatality rate is between $20 \%$ and $90 \%$ depending on the virus species $[10,11]$.

Zaire Ebola virus species has the highest mortality rate $(60 \%-90 \%)$ followed by the Sudan Ebola virus species $(40 \%-60 \%)$. The Bundibugyo virus species has caused only one outbreak to date, with a $25 \%$ mortality rate. In the current outbreak, when the first 9 months of data were reviewed case-fatality rate was reported as $70.8 \%$ [12]. Centers for Disease Control and Prevention (CDC) recommends that travelers to Guinea, Liberia, and Sierra Leone protect themselves by avoiding contact with the blood and body fluids of the diseased people. $\mathrm{Ni}$ geria, Cameroon, Central African Republic, Ghana, Angola, Togo, United Republic of Tanzania, Ethiopia, Mozambique, Burundi, Madagascar and Malawi are also at risk for spread of the EVD.

\section{Clinical manifestations}

EVD is an acute hemorrhagic fever, that has an incubation period of 2 to 21 days (mean 4-10) which is characterized by 'flu-like' symptoms like fever, chills, malaise, and myalgia. The febrile state may be mild during the initial phase of the illness, but may manifest with an acute, and very worse onset with associated chills and rigors. The most commonly described symptoms are fever in combination with a maculopapular rash around the face, neck, trunk, and arms usually appearing by day 5-7 of the illness. The most common symptoms were fever, fatigue, vomiting, diarrhea, and anorexia in current 
outbreak in Africa.

A person who has fever or symptoms as severe headache, fatigue, muscle pain, vomiting, diarrhea, abdominal pain, or unexplained hemorrhage; and an epidemiologic risk factor within the 21 days before the onset of symptoms must be kept under observation.

Viral hemorrhagic fever is the main finding. Because of thrombocythemiamucosal hemorrhage (especially in the conjunctiva), petechiae, ecchymosis, can be observed in patients with EVD. Massive hemorrhage, is usually observed only in fatal cases especially in the gastrointestinal system $[13,14]$. Other than the blood system, there are also cardiac, renal, pulmonary, neurological, gastrointestinal, and hepatic involvements. Cardiac manifestations might be due to the hemodynamic changes due to severe hemorrhage or direct viral involvement of the heart. But the cardiopathy in Ebola virus infection is not clear and needs further study. Profound fluid losses from the gastrointestinal tract result in volume depletion, metabolic abnormalities such as hyponatremia, hypokalemia, and hypocalcemia, shock, and organ failure. The patients who have respiratory, neurological, or hemorrhagic symptoms have higher risk of death [15]. Patients who survive from Ebola infection have been reported to show clinical improvement by the middle of the second week.

\section{Diagnosis}

EVD usually presents with an acute viral prodrome. It is difficult to distinguish EVD from other infectious diseases such as malaria, typhoid fever, meningitis, shigellosis, leptospirosis and yellow fever. Therefore, travel and exposure history is very important when approaching a suspected patient returning from an endemic area.

As laboratory findings, leukopenia with lymphocytes and an increased percentage of granulocytes are common signs at the time of clinical presentation As the disease progresses, leukocytosis can develop with an increase in immature granulocytes. Thrombocytopenia is a persistent feature until death. Mildly elevated serum levels of alanine and aspartate aminotransferase (ALT, AST) are common findings. Jaundice is not commonly seen, and serum total bilirubin level is either normal or elevated in the early phase of the illness. Elevated blood urea nitrogen and creatinine are observed due to excessive fluid loss from diarrhea and vomiting without adequate volume replacement. Prolonged prothrombin time (PT), partial thromboplastin time (PTT), or bleeding and disseminated intravascular coagulation (DIC) can be seen [16].

Antigen-capture enzyme-linked immunosorbent assay (ELISA) testing, IgM ELISA, polymerase chain reaction (PCR) and virus isolation tests are used as diagnostic tests to confirm infection. Laboratory diagnosis for EVD should be performed in a well-equipped laboratory with up to biosafety level 4 bio- contaminant facilities for viral culturing. WHO Risk Group 4 Pathogen requiring biosafety level 4-equivalent containment requiring Biosafety Level 4-equivalent containment, because of its high mortality rate.

There is no biosafety level 4 bio- contaminant facilities in Turkey so there is a need to establish a biosafety level-4 laboratory for further studies in Turkey.

\section{Case definitions for EVD}

According to the World Health Organization (WHO) and Centers for Disease Control and Prevention (CDC), a suspected case was defined as follows:

Any person, alive or dead, had or having a sudden onset of high fever and had contact with a patient who is suspected, probable or confirmed or a dead or sick animal from Ebola; or if any person with sudden onset of high fever with at least three of the following findings: severe headache, fatigue, anorexia/loss of appetite, lethargy, breathing difficulties, muscle pain, vomiting, diarrhea, abdominal pain, unexplained hemorrhage or any sudden death without any cause.

A probable case was defined as any suspected case who has had direct contact with the blood and body fluids of an individual diagnosed with Ebola or who has had close physical contact with an individual diagnosed with Ebola or who lived with or visited an Ebola-diagnosed patient while the patient was ill within the previous 21 days with no laboratory confirmation.

A confirmed case was defined as a suspected or probable case with a positive laboratory result. 
On 27 October 2014, the CDC updated the case definitions and included the category of "Person Under Investigation (PUI)" described as: A person who has fever or symptoms, including severe headache, fatigue, muscle pain, vomiting, diarrhea, abdominal pain, or unexplained hemorrhage; and an epidemiologic risk factor within the 21 days before the onset of symptoms.

A confirmed case was defined as a PUI with a positive laboratory confirmation of EBOV [17].

\section{Treatment}

The main strategies that is being offered to infected patients are symptomatic and supportive care, such as hydration, replacement of electrolytes, nutritional support, maintaining oxygen status and blood pressure and treating other infections. Currently there is no approved vaccine and standard treatment for EVD that has been validated in humans $[18,19]$.

Up to date there are two notable Ebola vaccine efforts. The first one is cAd3-ZEBOV and the second is the rVSV. Both vaccines are effective in nonhuman primates, but confirmation of effectiveness against the Ebola virus in humans requires further studies [20].

As a result of quick recovery of one of the Dallas (Texas USA) nurses following the transfer of plasma from Ebola survivors; passive immunity has become a potential treatment for EVD [21].

Because of the high mortality rate of EVD, many investigational treatments are underway. ZMapp, is an experimental biopharmaceutical drug under development for treatment of EVD. It contains three "humanized" monoclonal antibodies (mAbs) against the EBOV GP protein. These antibodies are produced in plants, specifically Nicotiana benthamiana [22].

This drug was first used experimentally in humans during the 2014 West Africa EVD outbreak, but there is no randomized controlled trial to determine whether it works or it is safe enough to bring it on the market [20, 23]. In early August 2014, "ZMapp", was used to treat two American medical workers and one Spanish priest and three African doctors who had contracted the deadly EBOV. Except for the 75-year-old Spanish priest who died despite the ZMapp treatment, the other five were reportedly improved significantly after receiving ZMapp. This is the first time that a drug has shown therapeutic efficacy against EVD in human.

Favipiravir is a new antiviral drug. It can be used for the treatment of patients with Ebola virus, but it is teratogenic, embryotoxic and any clinical trial of favipiravir has not been performed in patients infected with the Ebola virus. It is approved by The Minister of Health, Welfare and Labor of Japan due to the epidemic in West Africa [24].

Melatonin is a natural substance, which is synthesized at night by the pineal gland and intestinal cells among many other cell types. It has no known side effects, even at very high concentrations. Melatonin has significant and curative effects on all the relevant cells and systems and decreases the symptoms and increases the survival of those infected with the Ebola virus. It is expected that melatonin will help maximize the benefit of soon available treatments [25].

\section{Prevention}

Since Ebola virus infection is highly infectious and there are no standard treatments for EVD, isolation of infected individuals is very important. If a diagnosis of Ebola is being suspected, then, the patient should be isolated in a single room (with a private bathroom or covered bedside commode), and healthcare personnel should follow standard, contact, and droplet precautions, including the use of appropriate personal protective equipment (PPE). PPE should include double gloves, gown or coverall and apron, face mask, eye protection (goggles or face shield) head cover, and boots. when performing aerosol-generating procedures, the wearing N95 of or higher respirators is also suggested.

Before contact with patients, washing hands with soap and water or an alcohol-based hand sanitizer and wearing appropriate personal protective equipment are recommended. Once exposed to blood or body fluids of the unprotected patient, healthcare workers should thoroughly wash the exposure site with water and soap. Safety needles are recommended for venipuncture or blood samples.

A minimum volume of $4 \mathrm{~mL}$ of whole blood preserved with EDTA is preferred for Ebola testing. Specimens for shipment should be packaged following the basic triple packaging system that consists 
of a primary sealable container wrapped with $a b$ sorbent material, secondary container (watertight, leak-proof), and an outer shipping package and should be shipped at $2-8^{\circ} \mathrm{C}$ or frozen on cold-packs.

The defined case should be isolated till two negative results within a single 48 hour period are obtained. If death occurs from a suspect case of EVD, dead body should be burned within 24 hours.

In hospitals, transport and movement of the patients out of the room should be limited to medically-necessary purposes. If transport or movement outside the room is necessary, patients sould wear a surgical mask [26].

After returning from epidemic area, body temperature should be monitored for 21 days.

The Ebola virus is susceptible to many chemical agents. According to the WHO recommendations, environment contaminated with blood spill or body fluid can be cleaned up with 1:10 dilution and for surfaces $1: 100$ dilution of $5.25 \%$ household bleach applied for 10 minutes is required [25].

Conflict of Interest: No conflict of interest was declared by the authors.

Financial Disclosure: The authors declared that this study has received no financial support.

\section{REFERENCES}

1. Nanbo A, Watanabe S, Halfmann P, Kawaoka Y. The spatiotemporal distribution dynamics of Ebola virus proteins and RNA in infected cells. Sci Rep 2013;3:1206. CrossRef

2. Ebola haemorrhagic fever in Zaire, 1976. Bull World Health Organ 1978;56:271-93.

3 Ebola haemorrhagic fever in Sudan, 1976. Report of a WHO/International Study Team. Bull World Health Organ 1978;56:247-70.

4. Pinzon JE, Wilson JM, Tucker CJ, Arthur R, Jahrling PB, Formenty P. Trigger events: enviroclimatic coupling of Ebola hemorrhagic fever outbreaks. Am J Trop Med Hyg 2004;71:664-74.

5. Bagcchi S. Ebola haemorrhagic fever in west Africa. Lancet Infect Dis 2014;14:375. CrossRef

6. Maganga GD, Kapetshi J, Berthet N, Kebela Ilunga B, Kabange F, Mbala Kingebeni P, et al. Ebola virus disease in the Democratic Republic of Congo. N Engl J Med 2014;371:2083-91. CrossRef

7. Times TNY. Ebola Facts: How Many People Have Been Sent to Countries With Ebola by Doctors Without Borders? 2014. [Last accessed on 2014 Nov 15]. Available from: http://www. nytimes.com/interactive/2014/07/31/world/africa/ebola-viru- soutbreak-qa.html?_r=1

8. Chowell G, Simonsen L, Viboud C, Kuang Y. Is West Africa Approaching a Catastrophic Phase or is the 2014 Ebola Epidemic Slowing Down? Different Models Yield Different Answers for Liberia. PLoS Curr 2014;6. CrossRef

9. Centers for Disease Control and Prevention. 2014 Ebola Outbreak in West Africa - Case Counts. CDC; (Centers for Disease Control and Prevention). 2014.

10. Polonsky JA, Wamala JF, de Clerck H, Van Herp M, Sprecher A, Porten $\mathrm{K}$, et al. Emerging filoviral disease in Uganda: proposed explanations and research directions. Am J Trop Med Hyg 2014;90:790-3. CrossRef

11. World Health Organization. Ebola Hemorrhagic Fever. Geneva, Switzerland: WHO; 2010. Available at http://www. who.int/ mediacentre/factsheets/fs103/en/.

12. WHO Ebola Response Team. Ebola virus disease in West Africa--the first 9 months of the epidemic and forward projections. N Engl J Med 2014;371:1481-95. CrossRef

13. Kortepeter MG, Bausch DG, Bray M. Basic clinical and laboratory features of filoviral hemorrhagic fever. J Infect Dis 2011;204 Suppl 3:810-6. CrossRef

14. West TE, von Saint André-von Arnim A. Clinical presentation and management of severe Ebola virus disease. Ann Am Thorac Soc 2014;11:1341-50. CrossRef

15. Kortepeter MG, Bausch DG, Bray M. Basic clinical and laboratory features of filoviral hemorrhagic fever. J Infect Dis 2011;204 Suppl 3:810-6. CrossRef

16. Joffe S. Evaluating novel therapies during the Ebola epidemic. JAMA 2014;312:1299-300. CrossRef

17. Centers for Disease Control and Prevention. Case Definition for Ebola Virus Disease (EVD). Atlanta, GA, USA: CDC; 2014 Available at http://www.cdc.gov/vhf/ebola/hcp/casedefinition. html.

18. Kanapathipillai R, Henao Restrepo AM, Fast P, Wood D, Dye $\mathrm{C}$, Kieny MP, et al. Ebola vaccine--an urgent international priority. N Engl J Med 2014;371:2249-51. CrossRef

19. Butler D. Ebola drug trials set to begin amid crisis. Nature 2014;513:13-4. CrossRef

20. Bacon J, Owens M. Infected Dallas nurse ID'd; gets transfusion from Ebola survivor. 2014. 2014. Oct 28, [Last accessed on 2014 Oct 28]. Available from:http://www.usatoday.com/story/news/ nation/2014/10/13/ebola-nurse-who/17182599/

21. Zhang Y, Li D, Jin X, Huang Z. Fighting Ebola with ZMapp: spotlight on plant-made antibody. Sci China Life Sci 2014;57:987-8. CrossRef

22. Centers for Disease Control and Prevention. Ebola virus disease Information for Clinicians in U.S. Healthcare Settings. CDC USA Website. http://www.cdc.gov/vhf/ebola/hcp/clinician-information-us-healthcare-settings.html\#investigational-vaccines.

23. Nagata T, Lefor AK, Hasegawa M, Ishii M. Favipiravir: a new medication for the ebola virus disease pandemic. Disaster Med Public Health Prep 2015;9:79-81. CrossRef

24. Anderson G, Maes M, Markus RP, Rodriguez M. Ebola virus: 
melatonin as a readily available treatment option. J Med Virol 2015;87:537-43. CrossRef

25. Centers for Disease Control and Prevention. Ebola virus disease Information for Healthcare Workers and Settings. CDC USA Website. http:// www.cdc.gov/vhf/ebola/hcp/index.html.
26. Siegel JD, Rhinehart E, Jackson M, Chiarello L, and the Healthcare Infection Control Practices Advisory Committee, 2007 Guideline for Isolation Precautions: Preventing Transmission of Infectious Agents in Healthcare Settings http://www.cdc.gov/ ncidod/dhqp/pdf/isolation2007.pdf 